\title{
Disk-Resolved Photometry of Cometary Nuclei: Results from DIXI and Stardust-NExT
}

\author{
Jian-Yang $\mathrm{Li}^{1}$, Peter C. Thomas ${ }^{2}$, Joe Veverka ${ }^{2}$, \\ Michael F. A'Hearn ${ }^{3}$, Sebastien Besse ${ }^{3}$, Michael J. S. Belton ${ }^{4}$, \\ Tony L. Farnham ${ }^{3}$, Kenneth P. Klaasen ${ }^{5}$,Carey M. Lisse ${ }^{6}$, \\ Lucy A. McFadden ${ }^{7}$ and Jessica M. Sunshine ${ }^{3}$ \\ ${ }^{1}$ Planetary Science Institute \\ email: jyli@psi.edu \\ ${ }^{2}$ Cornell University, ${ }^{3}$ University of Maryland at College Park, ${ }^{4}$ Belton Space Exploration \\ Initiatives, LLC, ${ }^{5}$ Jet Propulsion Laboratory, California Institute of \\ Technology, ${ }^{6}$ JHU-APL,${ }^{7}$ NASA Goddard Space Flight Center
}

\begin{abstract}
Previous comet flyby missions enabled detailed studies of the photometric properties of several cometary nuclei from disk-resolved images, including 9P/Tempel 1, 19P/Borrelly, and 81P/Wild 2. Two recent missions, DIXI and Stardust-NExT, encountered Comets 103P/Hartley 2 and Tempel 1 respectively, expanding the pool of sampled cometary nuclei in their unique ways: Hartley 2 is a hyperactive comet; Tempel 1 was visited and impacted by the Deep Impact dualspacecraft during its previous perihelion passage. Photometric modeling shows that the global photometric properties of the nuclei of Hartley 2 and Tempel 1 are similar to those of other cometary nuclei. The photometric variation of the hyperactive nucleus of Hartley 2 is about $15 \%$, similar to that of weakly active comets Tempel 1 and Wild 2. The photometric properties of Tempel 1 measured by NExT suggest little change from those measured by DI. These results, together with the photometric properties of Wild 2 and Borrelly, indicate that the photometric properties of cometary nuclei are independent of the activity level and gross geomorphology of cometary nuclei. Instead, cometary nucleus photometric properties might be determined by its outgassing, which leaves low-albedo deposit on the surface and forms similar photometric texture. The time scale for the photometric alteration on cometary nuclei due to outgassing should be much shorter than the dynamic time scale.
\end{abstract}

